\title{
Micro- reactors and gas sensors based on locally heated carbon nanotubes decorated with Ti nanoparticles
}

\author{
R. Savu', J.V. da Silveira ${ }^{1}$,A. Alaferdov ${ }^{1}$, A. Flacker ${ }^{1,2}$, E. Joanni ${ }^{2}$, A.L. Gobbi ${ }^{3}$, M.A. Canesqui ${ }^{1}$, D. S. \\ de Lara ${ }^{1}$, A.L.P. Rotondaro ${ }^{2}$ and S.A. Moshkalev ${ }^{1}$ \\ ${ }^{1}$ Centro de Componentes Semicondutores - CCS, Universidade de Campinas - UNICAMP, C.P. \\ 6061, Rua João Pandia Calógeras, 90, 13083-870, Campinas, SP, Brasil. \\ stanisla@ccs.unicamp.br \\ ${ }^{2}$ Divisão de Microssistemas e Empacotamento, Centro de Tecnologia da Informação Renato Archer, \\ Rodovia D. Pedro I Km 143.6, 13069-901, Campinas, SP, Brasil. \\ ${ }^{3}$ Laboratório de Microfabricação, Laboratório Nacional de Luz Sincrotron - LNLS, Rua Giuseppe \\ Máximo Scolfaro 10.000, Pólo Il de Alta Tecnologia, Campinas, SP, Brasil.
}

\begin{abstract}
In this work, we report design and fabrication of micro-sensors and reactors based on nanoparticledecorated carbon nanotubes. The reactors, having a volume of $100 \mu \mathrm{l}$, were machined from one single Kovar $^{\Theta}$ piece in order to avoid leaks in the system due to additional welding. The connections for controlling pressure and atmosphere in the working chamber are provided by $1 / 4$ " Swagelok ${ }^{\circledR}$ tubes placed at diametrically opposite positions from the testing chamber. The contacts were electrically insulated from the metallic body of the reactor using borosilicate sealing glass frit. Successively, the reactor was gold plated and hermetically sealed using a lid with Viton ${ }^{\circledR}$ o-ring.

The dielectrophoresis technique was used for deposition of CNTs over patterned electrodes. Titanium adhesion layers $(30 \mathrm{~nm})$ and $150 \mathrm{~nm}$ gold films were sputtered onto $\mathrm{Si} / \mathrm{SiO}_{2}$ substrates. Sequentially, the gold layers were electrochemically thickened until $1 \mu \mathrm{m}$ and the electrodes were patterned using photolithography and wet chemical corrosion. Before the deposition, a gap $1 \mu \mathrm{m}$ wide and $5 \mu \mathrm{m}$ deep was milled in the metallic line by focused ion beam. This procedure allowed us to fabricate sensors based on suspended nanotubes bridging the electrodes. Successively, the sputtering technique was used for CNTs decoration with Ti nanoparticles. The as-obtained sensors were electrically connected to the reactor by the wire-bonding technique. The sensors were tested using different gases and pressure ranges. The small chamber volume allowed the measurement of fast sensor characteristic times, with the sensors showing good sensitivity towards gas as well as high reproducibility.
\end{abstract}

Key words: Micro-reactors, carbon nanotubes, decoration by nanoparticles, gas sensors.

\section{Introduction}

An important line of research in nanotechnology is related to carbon nanotubes (CNTs), due to their exceptional electrical, optical, mechanical and thermal properties [1-4]. These nanostructures are divided into two main categories: single-walled carbon nanotubes (SWCNTs) and multi-walled carbon nanotubes (MWCNTs) [1]. The decoration of CNTs with nanoparticles (CNTs/NPs), by means of chemical and/or physical methods, to produce novel hybrid structures proved to be a useful approach to further increase their range of applications. Therefore, carbon nanostructures, simple or decorated, are the basis of various novel micro- and nano-scale devices (ultra-fine probes, field effect transistors, sensors, etc.) [1, $2,4]$.

Micro-sensors in a chemiresistor configuration based on carbon nanotubes deposited precisely over metal electrodes from liquid solutions, using the ac dielectrophoresis process, have been recently reported $[5,6]$. The number of the nanotubes deposited by this method can be readily controlled by the process conditions, covering a range from several hundreds to an individual one. However, it should be noted that pristine nanotubes usually do not meet the basic gas sensor requirements of high and selective sensibility and short response and recovery times [7]. On the other hand, novel hybrid nanostructures, such as carbon 
nanotubes decorated with metal or metal oxide nanoparticles, proved to be very suitable for gas micro-sensing devices [5]. In this case, the functions important for gas sensing are separated between the two components as follows: 1) nanoparticles - interact strongly with the reactive gas injected in the test chamber, passing their electrical charges directly to the nanotubes; 2) carbon nanotubes - serving as templates, local heaters and low-resistance conductors (transducers). This separation of functions is important to provide a linear response of the sensor to detected gases, as the chemical interaction between gas and particles does not change the conductivity of nanotubes. Decoration of nanotubes by both metal and semiconductor nanoparticles has been used in our previous works to increase sensitivity and to decrease response times, as well as their selectivity to different gases $[5,6]$.

Micro-reactors that incorporate the above discussed sensors have the advantage of combining nanostructured sensing materials with small working volume, offering better monitoring and feature control and increasing functionality of the devices [8]. They allow sensor miniaturization and open the possibility of use in many new applications, such as very compact, non-invasive pressure sensors, accelerometers and gas sensors [7, 9].

In this work we report the design and fabrication of a micro-reactor that offers the advantages of easy assembly, low cost and good performance for testing a large variety of nano-devices. Moreover, the capsule easily fits to any vacuum/gas system and can be considered a mobile, easy to use, multiple tests chamber. The reactors were tested using a large number of sensors and proved to be highly suitable for testing carbon nanotube-based gas sensors.

\section{Experimental procedure}

A high vacuum system was design in order to allow the micro-sensors test using different pressures and gas composition, as can be observed in Fig. 1a. The system is composed by a central 6-way cross, a rotary and a turbo pump, wide range pressure sensors, mass flow controllers, valve and tubes (see Fig. 1b).

The reactors were designed in order to have a small volume and to fit to any vacuum/testing system. The test chamber (see Inset in Fig. 1b) is composed of a working cavity $(100 \mu \mathrm{l}$ volume), electric contact pins and an upper lid, all made in $\mathrm{Kovar}^{\circledR}$. Connections for controlling pressure and gas composition in the working chamber are provided by $1 / 4$ " Swagelok ${ }^{\circledR}$ tubes (1 $\mathrm{mm}$ internal diameter) placed at diametrically opposite positions. The body of the device was machined from a single metal piece, in order to avoid possible leaks in the system due to additional welding. The $0.5 \mathrm{~mm}$ Kovar $^{\circledR}$ pins were electrically insulated from the metallic body of the reactor using commercial borosilicate sealing glass frit thermally treated at $800^{\circ} \mathrm{C}$ in a nitrogen atmosphere. After this process was completed, the chamber was gold plated and the reactor was hermetically sealed using a lid with Viton ${ }^{\circledR}$ o-ring.

The gas sensors were fabricated by depositing CNTs, using the ac dielectrophoresis (DEP) method, onto oxidized silicon substrates with $1 \mu \mathrm{m}$ thick gold patterned electrodes. In order to obtain the metallic layers, $150 \mathrm{~nm}$ thick gold layers were sputtered over a $30 \mathrm{~nm}$ Ti adhesion layer and afterwards electrochemically thickened up to $1 \mu \mathrm{m}$. The patterning of the electrodes was made by photolithography followed by wet chemical etching. Before nanotubes deposition by DEP, from DMF (dimethylformamide) solutions, $1 \mu \mathrm{m}$ wide and $5 \mu \mathrm{m}$ deep gaps were milled across the metallic lines by a focused ion beam (NOVA 200 Nanolab - FEI Co.). The experimental parameters used for the DEP process were: a frequency of $100 \mathrm{kHz}$, a $3 \mathrm{~V}$ peak-to-peak voltage and a deposition time varying from 15 seconds to 3 minutes. In order to obtain hybrid nanostructures, the sputtering technique was employed for depositing thin $\mathrm{Ti}$ film over the carbon nanotubes. The deposition conditions used were: room temperature, $2 \times 10^{-2} \mathrm{mbar} \mathrm{Ar}$ pressure, 100W RF power and $6 \mathrm{~nm}$ film thickness. The as-obtained sensors were fixed in the working chamber and the electrical connections were made between the contact pads and the reactor pins using the wirebonding method (see Inset Fig. 1b).

The devices were characterized by field emission scanning electron microscopy (FESEM) and the electrical measurements were performed in pure oxygen and nitrogen atmospheres using mass flow controllers of 20sccm maximum flow (M100B - MKS Instruments) and a $2410 \mathrm{C}$ Keithley sourcemeter in the working power interval from 20 to $150 \mathrm{~mW}$. 


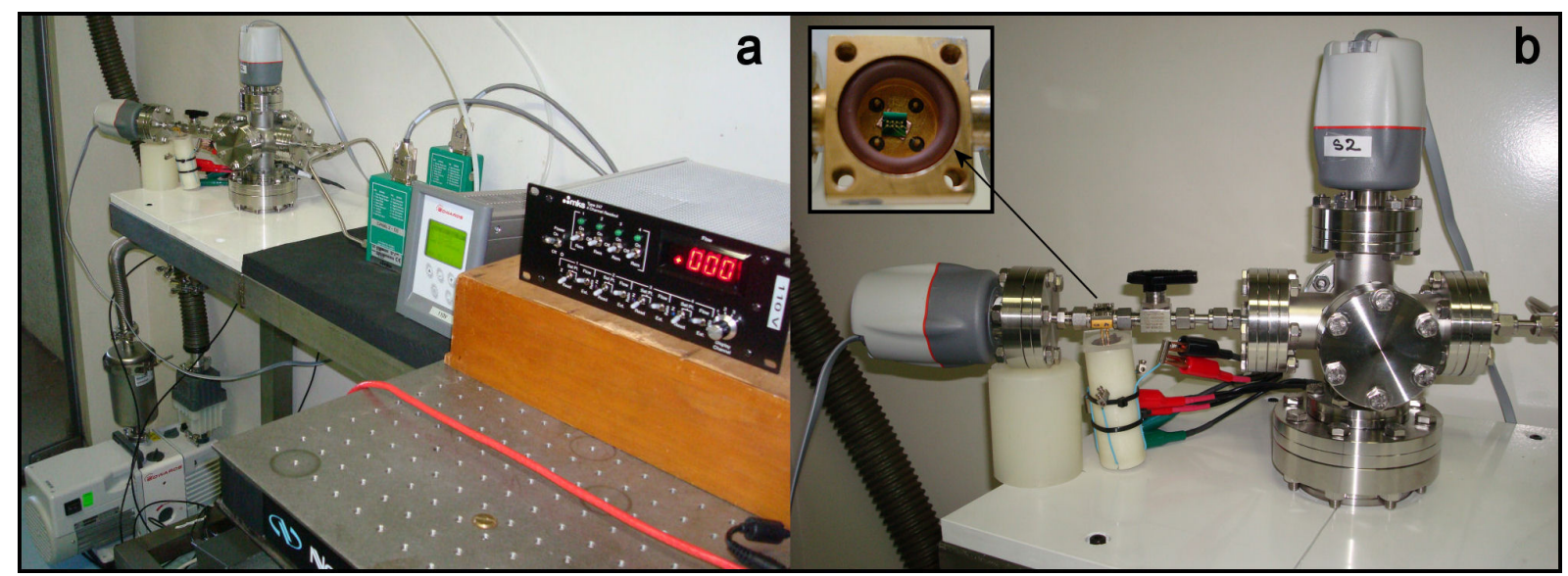

Fig. 1. High vacuum system designed for micro-reactor/sensor testing: a) general view showing its main parts and b) close view of a reactor coupled to this system. The Inset in Figure b illustrates an open micro-reactor encapsulating a chip with four sensors.

\section{Results and discussion}

Figure 2a presents the schematic drawing for sensor configuration (pristine or decorated CNTs) used in this work. The FE-SEM surface images of the gap milled between the electrodes (see Fig. 2b) show the gold electrodes, $1 \mu \mathrm{m}$ thick, deposited over oxidized silicon substrates. FE-SEM images of $\mathrm{Ti}$ decorated CNTs, with mean diameter of 30nm, deposited onto the gold electrodes and the results of Energy Dispersive Spectroscopy (EDS) measurements carried out on the central part of the area shown in the SEM image are presented in Figure 2c and 2d, respectively. For the device presented in Figure 2c, the deposition conditions were: a frequency of $100 \mathrm{kHz}$, a $3 \mathrm{~V}$ peak-to-peak voltage and a deposition time of 3 minutes.

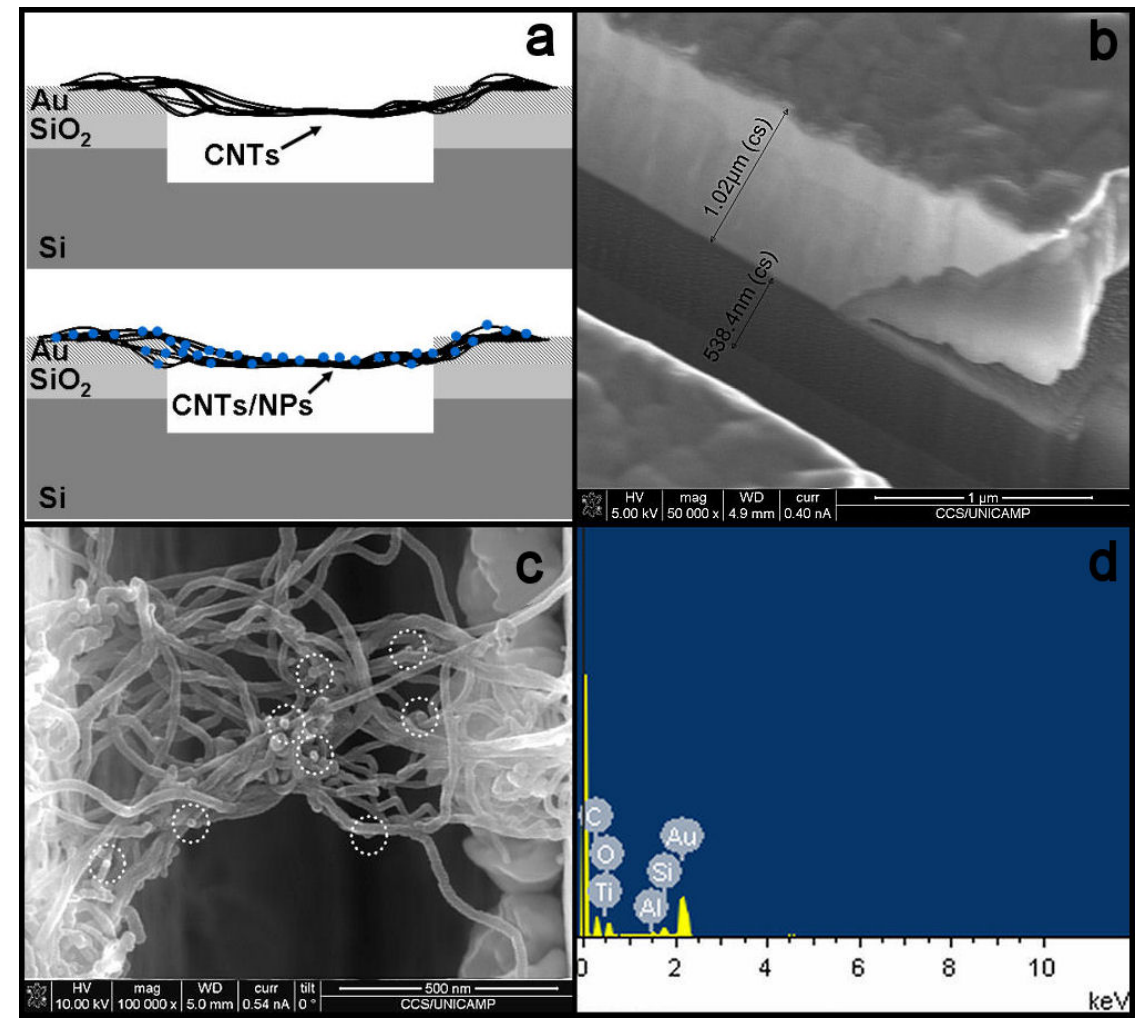

Fig.2. Schematic drawing of the suspended CNTs-based gas sensors (a); FE-SEM images of: the gap milled in the substrate by focused ion beam (b) and Ti decorated CNTs bridging the gap between the electrodes, with location of some metallic nanoparticles marked by circles (c); results of EDS measurements carried on the area presented in Figure $c(d)$. 
Subsequently, the device incorporating a network of suspended carbon nanotubes was introduced in the sputtering system and Ti was sputter deposited in order to increase the sensitivity of the sensors towards reactive gases, like oxygen. After the process with an RF power of $100 \mathrm{~W}$ and an argon pressure of $2 \times 10^{-2} \mathrm{mbar}$, the formation of a small number of separate metallic nanoparticles, with diameters up to $25 \mathrm{~nm}$, over nanotubes can be observed (see Fig. 2c). The EDS tests were performed in order to show that the weight percentage of $\mathrm{Ti}$ is near $3.1 \%$, confirming that quite small amount of Ti was deposited over nanotubes.

As one can observe in Figure 3, the sensitivity to $20 \mathrm{sccm}$ oxygen pulses for the device based on Ti-decorated carbon nanotubes is high. The device response to the gas injection is composed of two distinct phases: rapid and slow decays with characteristic times of $\sim 0.6 \mathrm{~s}$ and $\sim 4.5 \mathrm{~s}$, respectively. It is likely that the fast phase corresponds to the process of oxygen adsorption on the nanotubes and nanoparticles surfaces, followed by surface oxidation of $\mathrm{Ti}$ particles. The slower process probably is related to the oxygen diffusion within the particles followed by bulk Ti oxidation. It should be emphasized, that the characteristic times obtained here could not be measured in a conventional gas chamber where the chamber filling time usually exceeds $10 \mathrm{~s}$, as in a previous study $[5,6]$.

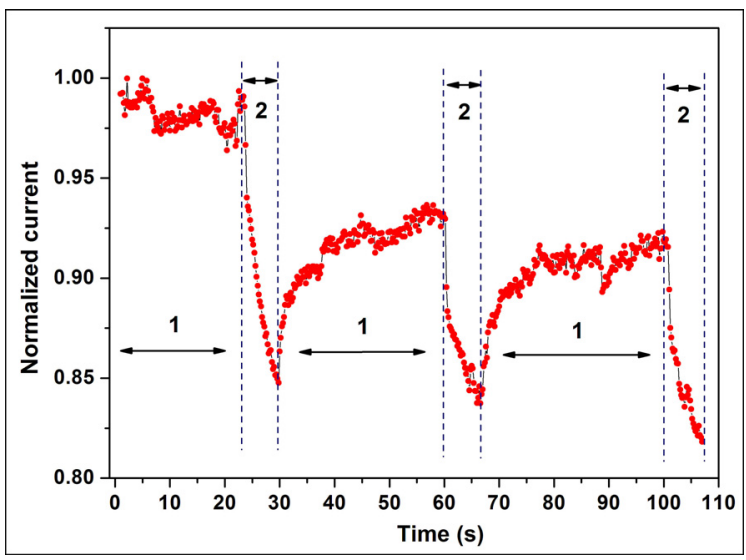

Fig. 3. Normalized current as a function of time for a sensor composed by CNTs decorated with Ti nanoparticles, submitted to three short cycles of vacuum (1) and $20 \mathrm{sccm} \mathrm{O}_{2}$ (2).

\section{Conclusions}

In conclusion, we designed and fabricated a $100 \mu \mathrm{l}$ volume reactor for testing the sensitivity of carbon nanotube-based sensors towards gases. Even though we tested gas microsensors, the reactor can be employed for testing a wide range of micro-devices. The design and the approach adopted for fabrication proved to be adequate for obtaining the desired small-volume atmosphere-controlled reaction chamber. One of advantages of our reactor lies in its simple assembly and ease of use with any vacuum/gas system. The four pins allow the connection of up to three devices fabricated on a single Si chip which can be independently tested by using one of the pins as a ground contact. Moreover, the lid can be modified in order to fit a window for performing in-situ optical characterization during device testing. The nanostructured sensors sealed in these reactors showed good sensitivity towards gas and pressure and high reproducibility. Due to reduced reactor volume, it was possible to measure a response time towards oxygen as short as $0.6 \mathrm{~s}$, with the sensor presenting high reproducibility and stability in the time interval used for the measurements.

\section{Acknowledgements}

J.V. Silveira and R. Savu would like to acknowledge the financial support of CAPES (grant no. 068/2007) and FAPESP (grant no. 2009/14600-4) Brazilian foundations, respectively. All the authors acknowledge the financial support of FAPESP and $\mathrm{CNPq}$ Brazilian foundations.

\section{References}

[1] K. Tanaka, T. Yamabe, K. Fukui, The Science and Technology of Carbon Nanotubes, Elsevier Science Ltd, Oxford, 1999.

[2] S. A. Wilson, et al., Materials Science and Engineering R 56, 1-129 (2007); doi: 10.1016/j.mser.2007.03.001.

[3] A. Loiseau, P. Launois, P. Petit, S. Roche, J.-P. Salvetat, Understanding Carbon Nanotubes, Lect. Notes Phys. 677, Springer, Berlin Heidelberg, 2006.

[4] Q. Cao, J. A. Rogers, Advanced Materials 21, 2953 (2009); doi: 10.1002/adma.200801995.

[5] R. V. Gelamo, F. P. Rouxinol, C. Verissimo, A. R. Vaz, M. A. Bica de Moraes, S. A. Moshkalev, Chemical Physics Letters 482, 302-306 (2009); doi: 10.1016/j.cplett.2009.10.019.

[6] R. V. Gelamo, F. P. Rouxinol, C. Verissimo, M. A. Bica de Moraes, S. A. Moshkalev, Sensor Letters 8, 488-492 (2010); doi: 10.1166/sl.2010.1299.

[7] P. Abgrall, N. T. Nguyen, Analytical Chemistry 80, 2326-2341 (2008); doi: 10.1021/ac702296u.

[8] W. C. Shin, R. S. Besser, Journal of Micromechanics and Microengineering 16, 731741 (2006); doi: 10.1088/0960-1317/16/4/009.

[9] 9 L. Dai, P. Soundarrajan, T. Kim, Pure and Applied Chemistry 74, 1753-1772 (2002); doi: 10.1351/pac200274091753. 